\title{
Could Anti-synthetase syndrome be a good predictor factor for interstitial lung disease?
}

\author{
Sineta Cristina Firulescu' ${ }^{1}$ Paulina Lucia Ciurea ${ }^{2}$, Cristina Dorina Parvanescu ${ }^{3}$, \\ Beatrice Andreea Chisalau', Andreea Lili Barbulescu ${ }^{4}$, Cristina Criveanu², \\ Adina Turcu-Stiolica ${ }^{5}$, Roxana Edmee Mustafa ${ }^{6}$, Florentin Ananu Vreju ${ }^{2}$ \\ ${ }^{1} \mathrm{PhD}$ student, University of Medicine and Pharmacy of Craiova, Romania \\ ${ }^{2}$ Department of Rheumatology, University of Medicine and Pharmacy of Craiova, Romania \\ ${ }^{3}$ Department of Rheumatology, Emergency County Hospital Craiova, Romania \\ ${ }^{4}$ Department of Pharmacology, University of Medicine and Pharmacy of Craiova, Romania \\ ${ }^{5}$ Department of Pharmacoeconomics, University of Medicine and Pharmacy of Craiova, Romania \\ ${ }^{6}$ Department of Cardiology, University of Medicine and Pharmacy of Craiova, Romania
}

\begin{abstract}
Anti-synthetase syndrome (SAS) is an autoimmune inflammatory myopathy, whose immune profile is dominated by anti-RNA synthetase antibodies (ARS), with a specific clinical phenotype. The major diagnostic criteria of SAS include the triad of myositis, arthritis and interstitial lung disease (ILD), on the background of positive anti-ARS antibodies. ILD in SAS, has a good evolution compared to the one in PM/DM, due to protective presence of anti Jo-1 antibodies.
\end{abstract}

Keywords: Anti-synthetase syndrome, anti-RNA, polymyositis, dermatomyositis

\section{INTRODUCTION}

Polymyositis and dermatomyositis (PM/DM) represent a group of inflammatory autoimmune myopathies (IIM), whose symp toms are including pain and weakness at the level of the proximal muscles associated with extramuscular involvement (1). Anti-synthetase syndrome is an autoimmune inflammatory myopathy, with a specific clinical phenotype and histological changes, whose immunological profile is dominated by anti-RNA synthetase antibodies (ARS) (2,3). The presence of anti-ARS antibodies in patients with IIM provides a predisposition for interstitial lung disease (ILD), which becomes a defining feature in terms of morbidity and mortality.

\section{CASE REPORT}

We present the case of a 68 -year-old patient with familial aggregation of autoimmune diseases (the mother with rheumatoid arthritis), who was diagnosed in 2015 with polymyositis, according to the Bohan and Peter criteria (1), and who, since 2015 until present, followed therapy with glucocorticoids
$10 \mathrm{mg} /$ day and azathioprine $100 \mathrm{mg} /$ day. In March 2016, the patient visited the emergency room (ER) for altered general condition, acute onset of dyspnea at low effort, tedious cough, poorly productive, proximal myalgia, with the onset 7 days before.

Clinical evaluation reveals the presence of muscle weakness, with painful and lightly swollen small joints of the hands, with peripheral vasospastic changes and pulmonary crepitation at bases. Besides this, the cardiovascular system, liver and spleen were in normal clinical range.

Biological and immunological assessment reveals increased muscle enzymes, antibody-specific antiJo1, in the setting of inflammatory biological syndrome. Cardiac evaluation by transthoracic cardiac Doppler ultrasound reveals an increased value of pulmonary arterial pressure (PAPs) - $32.2 \mathrm{mmHg}$ (with normal value $\leq 25 \mathrm{mmHg}$ ) (Fig. 1). It is decided to perform a high-resolution computer tomography (HRCT) chest scan that confirms the presence of pulmonary fibrosis with "ground glass" appearance (Fig. 2). 


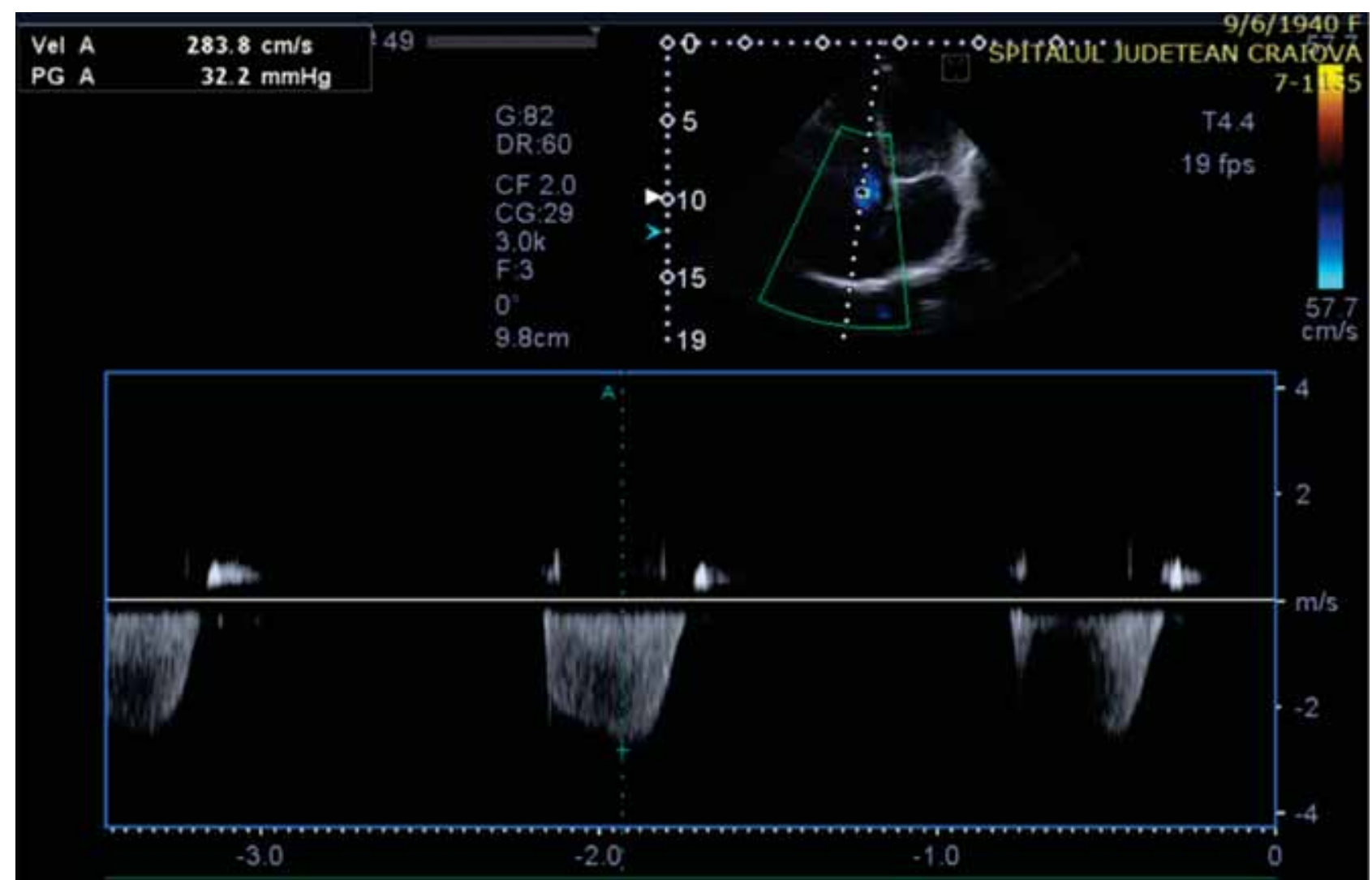

FIGURE 1. Cardiac evaluation by transthoracic cardiac Doppler ultrasound, showing a value of $32.2 \mathrm{mmHg}$ for PAPs

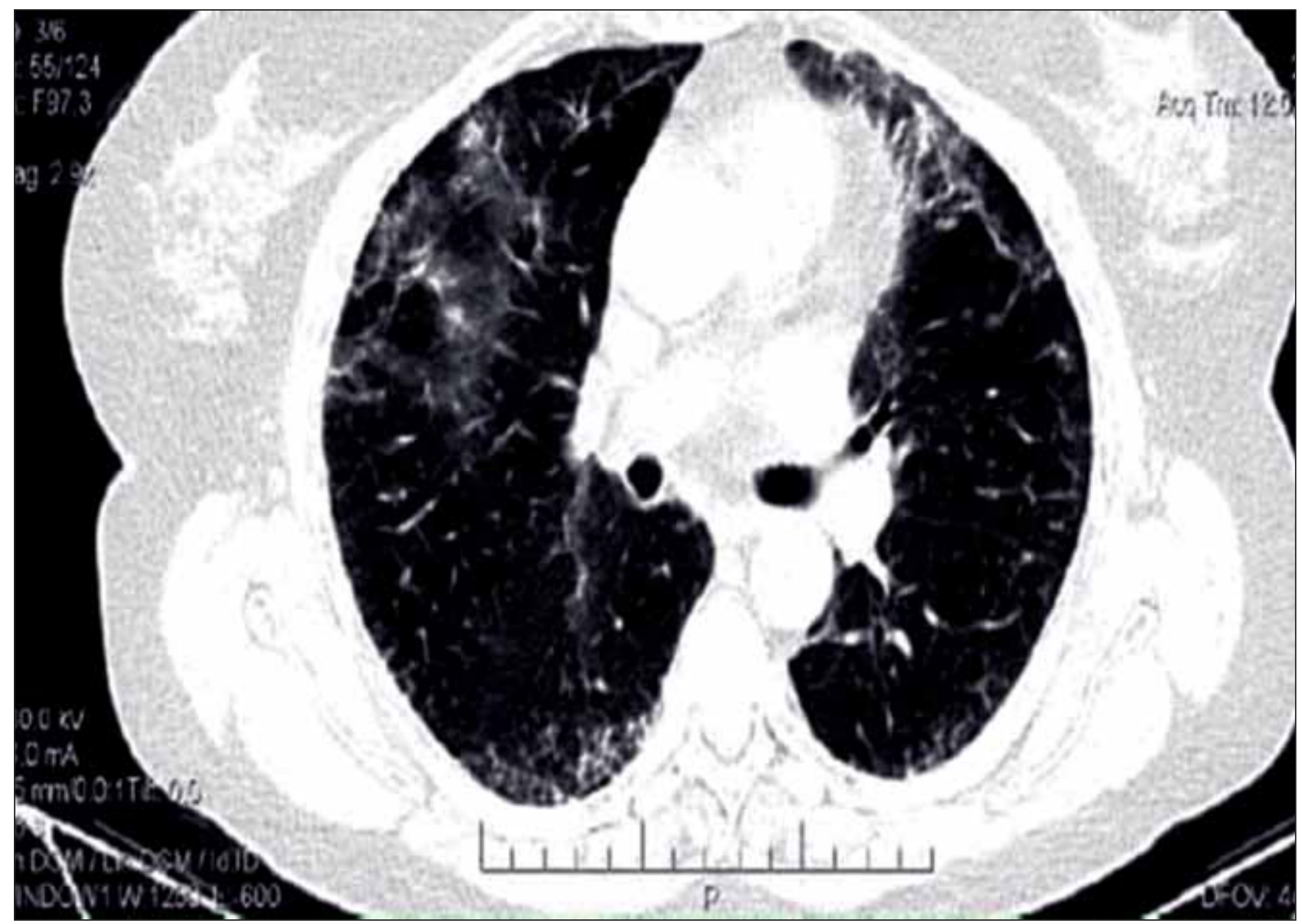

FIGURE 2. HRCT chest scan with pulmonary fibrosis with "ground glass" appearance 
The presence of clinical manifestations, in the setting of immunological and imagistic changes, confirmed the diagnosis of anti-synthetase syndrome (SAS).

Our patient received treatment with Cyclophosphamide $0.5 \mathrm{gm} / \mathrm{m}^{2}$, every 4 weeks for 6 months and then azathioprine $100 \mathrm{mg}$ /day as a maintenance therapy, with improvement of the pulmonary symptoms.

\section{DISCUSSIONS}

Polymyositis and dermatomyositis (PM/DM) are representing a group of inflammatory autoimmune myopathies (IIM), whose symptoms include pain and weakness of the proximal muscles associated with extramuscular changes, like cardiac or pulmonary involvement or swallowing dysfunctions (1).

In half $(50 \%)$ of the $\mathrm{PM} / \mathrm{DM}$ patients, we may find autoantibodies, but only a part of them are myositis-specific (MSA), being directed against components of a nucleosome remodeling complex (anti Mi2), a macromolecular complex that is involved in RNA processing (anti $\mathrm{PM} / \mathrm{Scl}$ ) or against ribonucleoproteins involved in protein synthesis, known as anti-synthetase antibodies (anti ARS).

The other antibodies that might be present in $\mathrm{PM} / \mathrm{DM}$ patients are just myositis associated (MAA) and could be found in varied autoimmune diseases.

Anti-synthetase syndrome is a specific subset of autoimmune inflammatory myopathy consisting of the presence of clinical features like Raynaud's phenomenon, arthralgia and fever, whose immunological profile is dominated by anti-RNA synthetase antibodies (ARS), having the interstitial lung disease (ILD) as a defining hallmark of the disease, in terms of morbidity and mortality (2). To note, that there are twenty types of anti ARS Abds, but eight of them were studied more and were associated with several important features: they are functionally directed against similar enzymes, they are associated with distinct phenotypes characterized by variation of manifestations of ILD, myositis and skin lesions and are mutually exclusive: anthistidil (Jo-1), threonil (PL-7), alanyl (PL-12), glycyl (EJ), isoleucil (OJ), Tyrosyl (Ha) and phenyl-alanyl-synthetase (Zo), directed to amino-acyl transferase RNA synthase (4).

Thus, patients usually have only one anti-ARS antibody and strong immunogenic associations have been described $(4,5)$.

Of these antibodies, anti-Jo1 antibodies are 4-5 times more frequent and they are described in
$15-20 \%$ of patients with myositis and in $70 \%$ of patients with ILD, while anti PL-7, anti-PL12 antibodies, appear in 2-3\% of patients with PM / DM and anti-OJ, EJ, KS antibodies in less than $2 \%$ patients with PM/DM. Though, compared to patients with anti Jo1 antibodies, the ones positive for anti-PL12 have a higher incidence of ILD (70-100\%).

Prevalence in epidemiological studies has not been studied due to the rare occurrence of this syndrome, but however some characteristics have been reported in low batches of patients, such as, early onset in life, around 18 years of age, women being particularly more affected, with a female: male report of 2.3: 1 (3).

The major diagnostic criteria for SAS include the triad of myositis, arthritis and ILD, on the background of positive anti-ARS antibodies. Fever, Raynaud's phenomenon and „mechanic's hands“ (MH) are considered minor manifestations or minor criteria, which can only support diagnosis and are not mandatory to be present, for establishing it. Presence of the three major criteria leads to the diagnosis of complete SAS, while the presence of just one or two major criteria, establishes the diagnosis of incomplete SAS. During the follow up period, the incomplete SAS may become complete, by the appearance of other major criteria. In incomplete SAS, the Raynaud's phenomenon, fever or MH are important because minor clinical manifestations may be predictive for subsequent occurrence of other major clinical manifestations $(6,7)$.

Lorenzo Cavagna et al. noticed that SAS with Jo-1 positive antibodies (Abds) is often presented as an incomplete form, being described at the onset in $80 \%$ of patients, especially in those with only one major clinical manifestation, with the subsequent development of new major changes. Thereby incomplete SAS with positive anti-Jo-1 Abds represents a phenotypic model, that worth monitoring, especially for ILD development $(6,8)$.

Although patients with SAS have common clinical symptoms, the presence of a specific anti-ARS antibody predisposes to a certain clinical outcome; thus, patients with anti-Jo-1 Abds present with myositis, the ones with anti-KS Abds present with ILD without myositis and patients with Anti-PL-7 Abds, with PM/DM-Scleroderma overlap syndrome. In Japanese patients, anti-PL-7 Abds are associated with ILD (9).

Most of the patients that were positive for antiJo-1, EJ, PL-7 antibodies were diagnosed at presen- 
tation with $\mathrm{PM}$ or DM, while the ones with $\mathrm{KS}$, OJ and PL-12 Abds were having ILD $(9,10)$.

Thereby our case was initially diagnosed as a polymyositis and after one year the patient developed incomplete SAS.

The most important symptom of SAS is interstitial lung disease. Prevalence of ILD, in patients with IIM, varies between 23 and $65 \%$, being the leading cause of death in these patients. Up to three quarters of PM/DM patients have ILD, which can be demonstrated by chest HRCT.

In relation to clinical features and prognostic, things are different between ILD in PM/DM, were the disease is rapidly progressive and steroid-resistant, compared to positive Anti-Jo-1 associated ILD, were Abds confer a protective course of disease and an increased patients' survival rate (11). Eighty-six percent of Jo-1 positive patients have ILD, and the presence of anti-ARS antibodies is predictive for late onset of myopathy and frequent recurrences of disease.

ILD onset may be acute with respiratory distress syndrome and respiratory failure, or progressive. The occurrence of inspiratory rales and pulmonary $\mathrm{x}$-ray changes (interstitial pattern) are characteristic for early ILD and requires spirometry and DLco diffusion, with confirmation by $\operatorname{HRCT}(12,13)$.

The treatment response is different in myositis related ILD compared to SAS related ILD and the treatment design incudes immunosuppressive agents, such as cyclophosphamide, azathioprine, mycophenolate mofetil, cyclosporine, tacrolimus, rituximab or intravenous immunoglobulins, along with glucocorticoids.

Cavagna L. observed, in a retrospective study which included 17 patients with positive Anti-Jo-1 Abds and ILD, that there is a low response on cyclosporine (Cyc) $3 \mathrm{mg} / \mathrm{Kgc} /$ day, in non-responder to cortisone patients. ILD was assessed by forced vital capacity, diffusion capacity and radiographic assessment.

Contrariwise, in the study of Kurita et al., which included 49 patients with myositis-associated ILD, the half of patients that received prednisolone plus cyclophosphamide or cyclosporine and had a poorer response compared to the other half receiving two immunosuppressants, such as cyclophosphamide or cyclosporine plus tacrolimus.

There are other studies on patients with positive ARS-Abds and refractory ILD to PDN and suppressive agents and who had improvement in pulmonary symptoms, demonstrated by HRCT, after one year of treatment with rituximab (12).

We can conclude, thus, that SAS patients with positive anti ARS antibodies tend to have a good response to glucocorticoids (GC) and Cyc, compared to ILD related myositis. Reduction of forced vital capacity (FVC), current volume (CV) and DLco, old age, symptomatic disease and resistance to $\mathrm{GC}$ are considered unfavorable prognostic factors. It was also noticed that SAS with anti Jo-1 positive Abds had a better prognosis and their presence seems to be a good predictor for survival compared to other antiARS antibodies at five (90\% vs. $75 \%)$ and ten years (70\% vs. $47 \%)(7,8,9,11,13)$.

\section{CONCLUSIONS}

As a conclusion, we can say that in patients with suspicion of PM/DM, HRCT and anti SAS antibodies should be performed in order to identify a possible ILD associated to antisynthetase syndrome. To mention that ILD in SAS, seems to have a good outcome compared to the one associated to $\mathrm{PM} / \mathrm{DM}$, due to protective presence of anti Jo-1 antibodies, but nonetheless, the pulmonary evaluation should be performed periodically in order to identify any major change and to optimize the treatment.

Conflict of interest: none declared Financial support: none declared

\section{REFERENCES}

1. Bohan A., Peter J.B. Polymyositis and Dermatomyositis (First of Two Parts). The New England Journal of Medicine. 1975, 292-17, 344-347.

2. Mahler M., Miller F.W., Fritzler M.J. Idiopathic inflammatory myopathies and the anti-synthetase syndrome: a comprehensive review. Review Autoimmun Rev. 2014, 13(4-5):367-71

3. Shinjo S.K., Levy-Neto M. Anti-Jo-1 antisynthetase syndrome. Bras J Rheumatol 2010; 50(5):492-500.

4. Labirua-Iturburu A., Selva-O'Callaghan A., Vincze M. et al. AntiPL-7 (Anti-Threonyl-tRNA Synthetase) Antisynthetase Syndrome
Clinical Manifestations in a Series of Patients From a European Multicenter Study (EUMYONET) and Review of the Literature. Medicine (Baltimore). 2012 Jul; 91(4):206-11

5. Aggarwal R., Cassidy E., Fertig N. et al. Patients with non-Jo-1 anti-tRNA-synthetase autoantibodies have worse survival than Jo-1 positive patients, Ann Rheum Dis. 2014 Jan; 73(1): 227-232.

6. Bartoloni E., Gonzalez-Gay M.A., Scirè C. et al. Clinical follow-up predictors of disease pattern change in anti-Jo1 positive antisynthetase syndrome: results from a multicenter, international and retrospective study. Autoimmun Rev. 2017 Mar; 16(3):253-257 
7. Solomon J., Swigris J.J., Brown K.K. Myositis-related interstitial lung disease and antisynthetase syndrome. J Bras Pneumol. 2011 Jan-Feb; 37(1):100-9

8. Cavagna L., Nuño L., Scirè C.A. et al. Clinical Spectrum Time Course in Anti Jo-1 Positive Antisynthetase Syndrome, Results from an International Retrospective Multicenter Study. Medicine (Baltimore). 2015 Aug; 94 (32)

9. Marie I., Josse S., Hatron P.Y. et al. Interstitial Lung Disease in Anti-Jo-1 Patients with Antisynthetase Syndrome, Arthritis Care Res (Hoboken). 2013 May; 65(5):800-8.

10. Hamaguchi Y., Fujimoto M., Matsushita T. et al. Common and Distinct Clinical Features in Adult Patients with Anti-AminoacyltRNA Synthetase Antibodies: Heterogeneity within the Syndrome, PLoS One. 2013; 8(4)
11. Hallowell R.W., Danoff S.K. Interstitial lung disease associated with the idiopathic inflammatory myopathies and the antisynthetase syndrome: recent advances. Curr Opin Rheumatol. 2014 Nov; 26(6):684-9

12. Chatterjee S., Prayson R., Farver C. Antisynthetase syndrome: Not just an inflammatory myopathy. Cleve Clin J Med. 2013 Oct; 80(10):655-66

13. Katzap E., Barilla-LaBarca M.L., Marder G. Antisynthetase Syndrome. Curr Rheumatol Rep. 2011 Jun; 13(3):175-81 